\title{
Towards a pure ZZ Ceti instability strip ${ }^{\star}$
}

\author{
B. G. Castanheira ${ }^{1,2}$, S. O. Kepler ${ }^{1}$, A. F. M. Costa $^{1}$, O. Giovannini ${ }^{3}$, E. L. Robinson ${ }^{2}$, D. E. Winget ${ }^{2}$, \\ S. J. Kleinman ${ }^{4}$, A. Nitta ${ }^{5}$, D. Eisenstein ${ }^{6}$, D. Koester ${ }^{7}$, and M. G. Santos ${ }^{1}$ \\ ${ }^{1}$ Instituto de Física, Universidade Federal do Rio Grande do Sul, 91501-900 Porto-Alegre, RS, Brazil \\ e-mail: barbara@if.ufrgs.br \\ 2 Department of Astronomy and McDonald Observatory, University of Texas, Austin, TX 78712, USA \\ 3 Departamento de Física e Química, Universidade de Caxias do Sul, Caxias do Sul, RS, Brazil \\ 4 Subaru Telescope, National Astronomical Observatory of Japan, 650 North A'ohoku Place, Hilo, HI 96720, USA \\ 5 Gemini Observatory, Northern Operations Center, 670 North A'ohoku Place, Hilo, HI 96720, USA \\ ${ }^{6}$ Steward Observatory, University of Arizona, 933 N. Cherry Ave. Tucson, AZ 85721, USA \\ 7 Institut für Theoretische Physik und Astrophysik, Universität Kiel, 24098 Kiel, Germany
}

Received 22 June 2006 / Accepted 15 September 2006

\section{ABSTRACT}

\begin{abstract}
Context. We have observed again two stars inside the ZZ Ceti instability strip that were previously classified as not-observed-to-vary (NOV) by Mukadam et al. (2004) and found them to be low-amplitude variables. Some evidence points to a pure ZZ Ceti instability strip; other evidence contests it.

Aims. The two stars previously classified as NOV have Sloan Digital Sky Survey (SDSS) spectroscopic effective temperatures that place them inside the ZZ Ceti instability strip, and they were "contaminating" the strip as constant stars, which could indicate that the instability strip was no longer a simple evolutionary stage. A pure instability strip indicates that pulsation is a normal phase which all DAs must go through.

Methods. We used effective temperatures derived from SDSS optical spectra by comparing them with model atmospheres to look for pulsators through time-resolved photometry and stars previously classified as NOV.

Results. Our new results indicate, but do not prove, a pure instability strip, because there are still other NOV stars that need to be observed again. Additionally, we have discovered five other ZZ Ceti stars based on their effective temperatures.
\end{abstract}

Key words. stars: white dwarfs - stars: variables: general - stars: oscillations

\section{Introduction}

The observational DA instability strip (ZZ Cetis) ranges in effective temperature $\left(T_{\text {eff }}\right)$ from 12270 to $10850 \mathrm{~K}$ (Gianninas et al. 2005; Mukadam et al. 2004). The current best theoretical models (Fontaine et al. 2001; Bradley 1996; Arras et al. 2006) predict that the ZZ Ceti instability strip should be pure: all the DA white dwarf stars, with temperatures within the instability strip limits should pulsate, with a small dependency on mass. The theory agreed extremely well with observations until the search for new pulsators was extended to white dwarfs discovered by the Sloan Digital Sky Survey (SDSS), which reaches a fainter population $(16<g<20)$ than the old sample. Using accurate temperature determination either from optical or ultraviolet spectra, the instability strip using only the old bright sample did not contain any non pulsator (e.g. Bergeron et al. 2004; Gianninas et al. 2005).

However, in the searches conducted by Mukadam et al. (2004) and Mullally et al. (2005), a substantial fraction of the DA white dwarf stars with temperatures placing them inside the instability strip do not show pulsation above their detection limit, so were called not-observed-to-vary (NOV). Many questions were raised based on these new observations. Is the instability strip pure? Which are the physical mechanisms that can

^ Partially based on observations at Observatório do Pico dos Dias/LNA; the Southern Astrophysical Research telescope, a collaboration between CNPq-Brazil, NOAO, UNC, and MSU; and McDonald Observatory of The University of Texas at Austin. prevent pulsation? What does this particular sample have that is different from the old one? Can the $\mathrm{H}$ layer be extremely thin so that pulsations will not happen? Can even weak magnetic fields prevent pulsation? Do the NOVs have unfavorable viewing angles?

If the instability strip is really contaminated with non pulsators, the models for DA white dwarfs and the results derived from these models need to be revised. Before taking such a crucial but drastic step, it is important to make sure the observational results are correct. There are several ways to falsify a contaminated instability strip. First, the temperatures of the SDSS sample are derived from spectra with a signal-to-noise rate $(S N R)<35$. In this scenario, the temperatures and masses of the non variables could simply be inaccurate and the stars actually lie outside the instability strip (Gianninas et al. 2005). There should also be some pulsators outside the strip due to these uncertainties, and the searches should be extended to a wider range of temperatures (e.g. the odd pulsator WDJ 2350-0054 at $10350 \mathrm{~K}$ in Mukadam et al. 2004). Second, pulsating white dwarf stars can appear to be extremely low-amplitude pulsators for a few hours due to the beating of many excited modes (Kleinman et al. 1998). A third but not the least important possibility is that variations could have been, in fact, smaller than the published detection limits, which vary from 2 to 9 mma (e.g. Kanaan et al. 1992).

The only way to solve the question about the purity of the ZZ Ceti instability strip is to observe these stars again through 
Table 1. Journal of observations with 2.1-m telescope at McDonald Observatory, 4.1-m at SOAR, and 1.6-m at OPD.

\begin{tabular}{cccccc}
\hline \hline Star & Run start (UT) & $t_{\text {exp }}(\mathrm{s})$ & $\Delta T(\mathrm{~h})$ & \# Points & Telescope \\
\hline SDSS J000006.75-004654.0 & $2005-08-0407: 29$ & 45 & 2.5 & 199 & $1.6-\mathrm{m}$ \\
& $2005-08-0605: 23$ & 45 & 1.6 & 127 & $1.6-\mathrm{m}$ \\
& $2005-08-0705: 53$ & 50 & 1.2 & 88 & $1.6-\mathrm{m}$ \\
SDSS J030325.22-080834.9 & $2005-12-0502: 59$ & 30 & 3.0 & 301 & $4.1-\mathrm{m}$ \\
& $2005-12-0602: 29$ & 30 & 3.5 & 347 & $4.1-\mathrm{m}$ \\
& $2005-12-0701: 10$ & 30 & 4.0 & 400 & $4.1-\mathrm{m}$ \\
SDSS J085325.55+000514.2 & $2005-12-0806: 10$ & 30 & 3.3 & 229 & $4.1-\mathrm{m}$ \\
& $2005-12-0909: 50$ & 30 & 3.5 & 170 & $4.1-\mathrm{m}$ \\
SDSS J085507.29+063540.9 & $2005-12-1105: 02$ & 30 & 2.8 & 276 & $4.1-\mathrm{m}$ \\
SDSS J091635.07+385546.2 & $2006-01-0706: 35$ & 5 & 1.3 & 921 & $2.1-\mathrm{m}$ \\
& $2006-01-0808: 46$ & 5 & 1.4 & 969 & $2.1-\mathrm{m}$ \\
SDSS J165020.53+301021.2 & $2005-08-0200: 37$ & 40 & 2.2 & 191 & $1.6-\mathrm{m}$ \\
SDSS J233458.71+010303.1 & $2005-08-0606: 14$ & 50 & 2.2 & 306 & $1.6-\mathrm{m}$ \\
& $2005-08-1200: 36$ & 30 & 1.5 & 198 & $4.1-\mathrm{m}$ \\
\hline
\end{tabular}

Notes: $\Delta T$ is the total length of the each observing run and $t_{\exp }$ is the integration time of each exposure.

higher $S N R$ spectroscopy and time-resolved photometry. The higher $S N R$ spectra allow a more accurate determination of $T_{\text {eff }}$ and $\log g$ (Kepler et al. 2006). In this paper, we present our first results on the re-observations of the time-resolved photometry of two stars classified as NOVs, finding variability in their light curves. As every NOV we observed is in fact a lowamplitude variable, we show strong observational evidence of a pure ZZ Ceti instability strip.

\section{Observations}

We observed our target list with the SOAR 4.1-m telescope, using the SOAR Optical Imager, a mosaic of two EEV $2048 \times$ 4096 CCDs, thinned and back-illuminated, with an efficiency around $73 \%$ at $4000 \AA$, at the Nysmith focus. We observed in fast readout mode with the CCDs binned $4 \times 4$, to decrease the readout+write time to $6.4 \mathrm{~s}$ and still achieve a $0.354^{\prime \prime} /$ pixel resolution. The exposure times were $30 \mathrm{~s}$, and we observed up to $4 \mathrm{~h}$. All observations were obtained with a Johnson $B$ filter to maximize the amplitude and minimize the red fringing.

We also observed with the Otto Struve 2.1-m telescope at McDonald Observatory, using the Argos camera. This camera is mounted at the prime focus of the telescope, it has a frame transfer CCD, and uses a red cutoff BG40 filter to reduce the scattered light from the sky (Nather \& Mukadam 2004). The integration times were $5 \mathrm{~s}$ and the observations spam for up to $1.4 \mathrm{~h}$.

Extending our search for new pulsators, we used the 1.6-m telescope at Observatório do Pico dos Dias, LNA, in Brazil. For these observations, we used a frame transfer CCD, with a quantum efficiency of $60 \%$ around $4000 \AA$. We observed with no filters because the CCD used has no detectable fringing and the g-mode pulsations in ZZ Ceti stars are coherent at all optical wavelengths (Robinson et al. 1982). The integration times were from 40 to $50 \mathrm{~s}$ and spanned up $2.5 \mathrm{~h}$. In Table 1, we show the journal of observations.

For each telescope, we chose the filter set up for which the $S N R$ were optimal.

The technique used to detect variability is differential time series photometry, comparing the targets with the other stars in the same field to minimize the effects of sky and transparency fluctuations. For each run, we extracted light curves using the hsp scripts developed by Antonio Kanaan for IRAF, with weighted aperture photometry, using different aperture sizes and selecting the light curve with the highest $S N R$ in the Fourier transform.

For the stars previously classified as NOVs, we had to observe them for a minimum of three hours. This minimum time was chosen to avoid destructive beating between modes (if there are any that are excited in the star) and to be able to reach a lower amplitude limit than previous observations. The NOVs we observed had detection limits above 3-4 mma.

In order to have some objective criterion for determining which peaks are real in the discrete Fourier transform, we adopted an amplitude limit such that a peak exceeding this limit only has a $1 / 1000$ probability of being due to noise (false alarm probability). For these continuous data sets, peaks above $3\langle A\rangle$ ( 3 times the square root of the average power) have a probability smaller than 1 in 1000 of being noise. We checked the detection limit for each star by Monte Carlo simulation, randomizing the acquisition times.

To know if another peak in the Fourier transform is an intrinsic periodicity of the star or whether it is only due to the spectral window, we subtracted from the light curve, the sine curve with the same amplitude, period, and phase information as the peak selected in the Fourier transform. After subtracting it from the light curve, we recalculated the Fourier transform to verify whether the sine curve was correct. Then, we repeated the procedure for the remaining periodicities.

\section{New variables}

The stars SDSS J030325.22-080834.9 and SDSS J085325.55+ 000514.2 were listed in Mukadam et al. (2004) as NOV objects with detection limits of 3-4 mma. In Fig. 1, we show the Fourier transform of the new pulsators previously classified as constant stars, both being low-amplitude pulsators, with amplitudes below the previous detection limit.

We show in Fig. 2 the Fourier transforms of the total light curves of the new stars discovered to be pulsators that have never been searched before. These stars were incorporated in our candidate target list based on the same criterion: their $T_{\text {eff }}$, derived from fitting the SDSS optical spectra to Koester's synthetic spectra derived from model atmospheres. We used a model atmosphere grid similar to the one described by Finley et al. (1997), but larger and denser. Within the $T_{\text {eff }}$ range from 11000 to $12000 \mathrm{~K}$, we have a $90 \%$ probability of finding pulsators (e.g. Mukadam et al. 2004). 
Table 2. Physical parameters derived by comparing the whole SDSS optical spectra with a grid of model atmospheres and SDSS magnitudes.

\begin{tabular}{cccccc}
\hline \hline Star & $T_{\text {eff }}(\mathrm{K})$ & $\log g$ & $g(\mathrm{mag})$ & Mass $\left(M_{\odot}\right)$ & $T_{\text {eff }}(\mathrm{K})(\langle P\rangle)$ \\
\hline WDJ 0000-0046 & $10880 \pm 110$ & $8.32 \pm 0.09$ & 18.84 & $0.81 \pm 0.06$ & 11310 \\
WDJ 0303-0808 $^{1}$ & $11400 \pm 110$ & $8.49 \pm 0.06$ & 18.74 & $0.92 \pm 0.04$ & 10960 \\
WDJ 0853+0005 & $11750 \pm 110$ & $8.11 \pm 0.06$ & 18.23 & $0.68 \pm 0.04$ & 11710 \\
WDJ 0855+0635 & $11050 \pm 50$ & $8.43 \pm 0.03$ & 17.25 & $0.88 \pm 0.02$ & 11140 \\
WDJ 0916+3855 & $11410 \pm 50$ & $8.10 \pm 0.03$ & 16.56 & $0.67 \pm 0.02$ & 11410 \\
WDJ 1650+3010 & $11100 \pm 90$ & $8.76 \pm 0.08$ & 18.11 & $1.07 \pm 0.04$ & 11620 \\
WDJ 2334+0103 & $11400 \pm 210$ & $7.99 \pm 0.14$ & 19.24 & $0.60 \pm 0.08$ & 10930 \\
\hline
\end{tabular}

Notes: we derived the masses using $T_{\text {eff }}$ and $\log g$ obtained from optical spectra and comparing these values with C/O white dwarf evolutionary models (Wood 1995). We also calculated the $T_{\text {eff }}$ using its relationship with weighted mean pulsation period (see Table 3) derived by Mukadam et al. (2006). The quoted uncertainties are the internal uncertainties due to fitting procedures only. ${ }^{1}$ Values derived from Gemini spectra are $T_{\text {eff }}=$ $11960 \pm 160$ and $\log g=8.305 \pm 0.017$ using LPT fit and $T_{\text {eff }}=11420 \pm 110$ and $\log g=7.821 \pm 0.016$ when the fit procedure included the whole spectrum (Kepler et al. 2006).

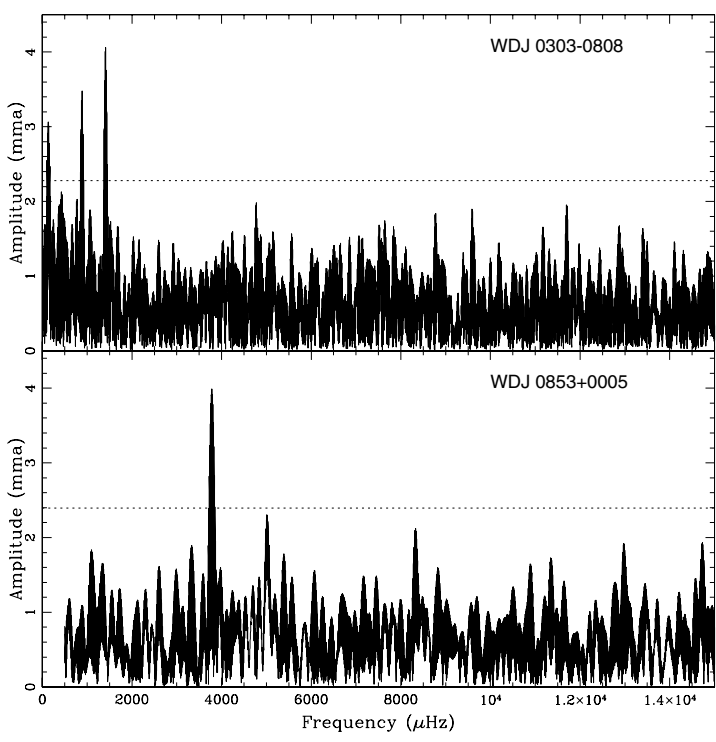

Fig. 1. Fourier transform of all the data for the stars previously classified as NOV WDJ 0303-0808 and WDJ 0853+0005. The dotted lines represent the detection limit above $3\langle A\rangle$, for which peaks have a $1 / 1000$ probability of being due to noise.

Table 3. Periodicities detected in our discovery runs for the new ZZ Ceti stars.

\begin{tabular}{ccc}
\hline \hline Star & Period $(\mathrm{s})$ & Amplitude $(\mathrm{mma})$ \\
\hline WDJ 0000-0046 & $611.42 ; 584.84 ; 601.35$ & $23.00 ; 15.92 ; 8.97$ \\
WDJ 0303-0808 & $707 ; 1128$ & $4.1 ; 3.5$ \\
WDJ 0853+0005 & 264.35 & 3.99 \\
WDJ 0855+0635 & $850 ; 433$ & $44 ; 15$ \\
WDJ 0916+6108 & $485.09 ; 447.70 ;$ & $32.89 ; 14.44 ;$ \\
& $238.10 ; 747.20$ & $10.76 ; 9.06$ \\
WDJ 1650+3010 & 339.06 & 14.71 \\
WDJ 2334+0103 & 923.15 & 40.37 \\
\hline
\end{tabular}

Table 2 shows a summary of the physical properties of the new pulsators derived by fitting the optical spectra up to $7000 \AA$ to Koester's models in the same way as described in Kleinman et al. (2004) and Eisenstein et al. (2006). We measured the $\chi^{2}$ on the difference between the observed spectra and the models, allowing for a reflux of the models according to a low-order polynomial. This step was made to correct for effects of unknown reddening. We used the first seven Chebychev polynomials in a linear wavelength as our refluxing basis. The uncertainties quoted in the table were estimated as the internal uncertainty

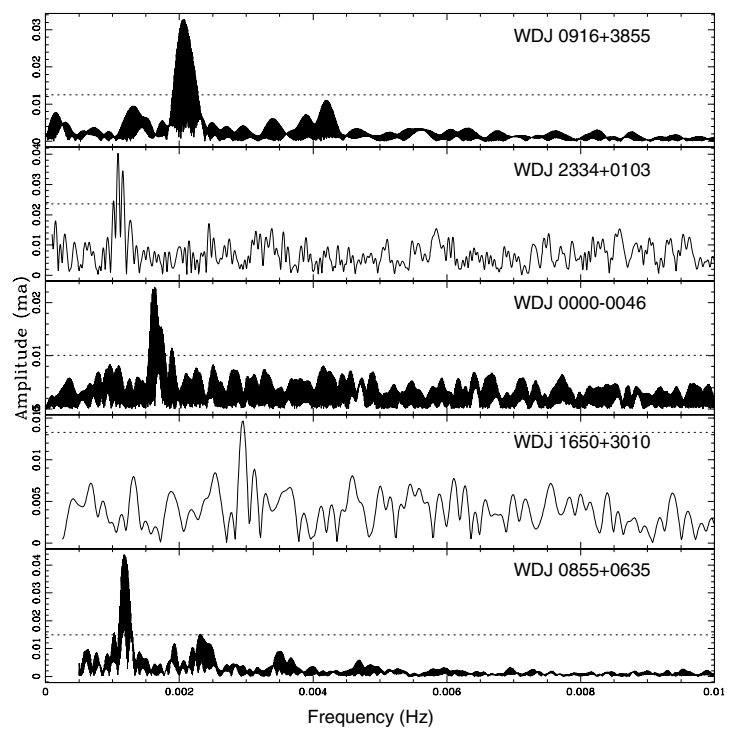

Fig. 2. Fourier transform of our search for new pulsators using the telescopes 2.1-m at McDonald Observatory, 1.6-m at Observatório do Pico dos Dias, and the $4.1-\mathrm{m}$ at SOAR. The candidates were selected by their $T_{\text {eff }}$ derived from comparison of optical spectra with model atmospheres. The dotted lines represent the detection limit above $3\langle A\rangle$, for which peaks have a $1 / 1000$ probability of being due to noise.

of the fit only, not considering that $T_{\text {eff }}$ and $\log g$ are correlated. These estimates also do not consider the changes in $T_{\text {eff }}$ during the pulsation (see Sect. 4.1 for a detailed discussion) or external uncertainties, which are dominant.

We analyzed the light curves of each one of the new pulsators to identify their periodicities, necessary in future seismological analysis for studying their properties and structure. The list of detected periods in these stars is shown in Table 3.

\section{Discussions}

\subsection{Purity of the instability strip}

In Fig. 3 we show the updated SDSS ZZ Ceti instability strip, including the NOVs reported by Mukadam et al. (2004) and Mullally et al. (2005) that still need to be re-observed.

At this point it is necessary to discuss the star WDJ 2350-0054, which has a published $T_{\text {eff }}=10350 \pm 60$ (Mukadam et al. 2004), indicated by an asterisk in Fig. 3. It is a pulsating star outside the instability strip with $T_{\text {eff }}$ significantly cooler than the red edge. Because there is no reasonable 


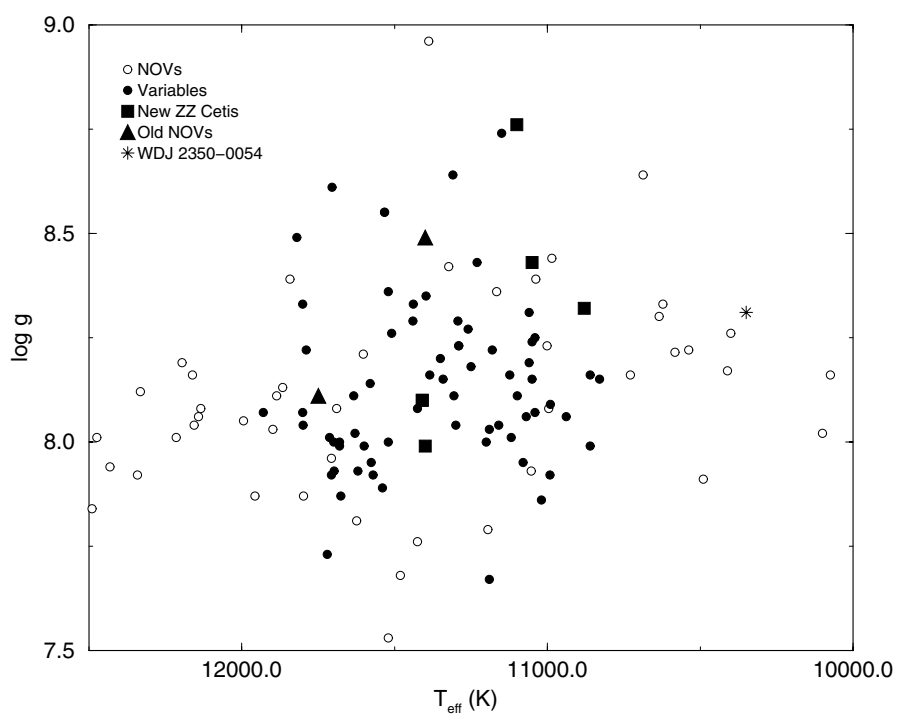

Fig. 3. Observational instability strip containing only SDSS stars observed for variability. The filled circles represent the ZZ Cetis and the open circles the stars for which variation in the light curve was not observed (NOVs) in previous works that need to be re-observed. The filled squares are the new ZZ Cetis reported in this paper, and the filled triangles are the stars that were previously classified as NOVs, but we detected variability. The asterisk marks a variable star that, according to SDSS $T_{\text {eff }}$, is outside the ZZ Ceti instability strip.

explanation for such a low temperature, we will not consider this star as the red edge of the instability strip. There are three independent SDSS spectra for this star, all of them providing a lower $T_{\text {eff }}$ than does the red edge when the whole spectrum is used in the fitting procedure. However, our line profile technique (LPT) fit, which has a larger scatter in the $T_{\text {eff }}$ determination for the SDSS spectra because of the lower $S N R$ in the lines, the resulting $T_{\text {eff }}$ brings this particular star inside the instability strip. High SNR spectra are necessary to reliably determine its physical parameters.

We detected variability in two stars previously classified as NOVs. There are still 19 NOVs inside the instability strip, which need to be observed again. A strong argument for a pure instability strip is that every star we re-observed for variability turned out to be a pulsator.

Kepler et al. (2005) discuss the external uncertainty measured from SDSS duplicate spectra, showing the uncertainty is around $300 \mathrm{~K}$ in $T_{\text {eff }}$ and $0.21 \mathrm{dex}$ in $\log g$, a significant portion of the size in $T_{\text {eff }}$ of the instability strip $(\sim 1400 \mathrm{~K})$. There is also another important effect on the contamination of a pure instability strip with non-pulsators: temperature variation during a pulsation cycle. For a low-amplitude low $\ell$ pulsator, the changes should be rather small, around $50 \mathrm{~K}$, but for larger amplitude pulsators, temperature variation can reach $500 \mathrm{~K}$ (Robinson et al. 1982).

The Gianninas et al. (2005) simulation of an LPT fitting shows that uncertainties for the SDSS spectra are on the order of $450 \mathrm{~K}$ for the average $S N R \sim 20$ in our sample. These are external uncertainties of the LPT method; however, we used the whole spectra fitting.

Kepler et al. (2006) report results from Gemini spectra with SNR around 100 for four stars from the SDSS sample. They show that their new $T_{\text {eff }}$ were similar to the ones obtained with SDSS spectra. The SDSS quoted uncertainties are underestimated by $60 \%$ in $T_{\text {eff }}$ and by a factor of 4 in $\log g$, and the correlation between $T_{\text {eff }}$ and $\log g$ is an important factor. Their simulation indicates that fitting using the whole spectra with reliable flux calibration is better than only an LPT fit.

All these uncertainties added together can falsify our interpretations of the impurity of the instability strip. Higher SNR spectra averaging out pulsations (e.g. Bergeron et al. 2004), as well as longer exposure time-series imaging, are required for lowering the uncertainties in temperature and the detection limits of pulsations for all stars within the boundaries of the ZZ Ceti instability strip.

\subsection{Changes in pulsation spectrum with temperature}

While the star cools down inside the instability strip, its pulsation spectrum changes, as do the pulsation amplitudes. Evolving from the blue (hot) to the red (cold) edge, the periods get longer and their amplitudes larger. For example, the hottest known pulsator, G 226-29 (Gianninas et al. 2005), has a dominant mode at $109 \mathrm{~s}$ and an amplitude of around 3 mma (Kepler et al. 1995) in the optical and no other periodicities besides the components of this triplet. On the other hand, the cooler star G 29-38 has 20 identified modes (Kleinman et al. 1998), and its pulsation spectra change dramatically from run to run. In one season, this star showed low amplitude pulsation; but its dominant mode is normally around $700 \mathrm{~s}$, and the pulsation amplitude for a single mode can reach $60 \mathrm{mma}$. All five of the new variables presented in Fig. 2 are long-period high-amplitude pulsators, while the previous NOVs have low amplitude, independent of their periods.

The qualitative explanation for the change in pulsation spectra within the ZZ Ceti instability strip is based on the behavior of the convection zone, starting at the base of the partial ionization layer, which moves inwards as the star cools. The convection zone gets bigger, allowing more modes to be excited, as the star progresses to the red edge. Because the base of the convection zone is deeper, the thermal time is longer, so the excited periodicities are rather long. In addition, as the position of the convection zone is more internal for cooler stars, more energy is needed to move the upper layers, which leads to higher amplitudes. Note that the timescale for crossing the instability strip is almost 1 Gyr.

Even though we understand how pulsations start, because of the increase in opacity at the developing partial ionization zone, we do not understand how the pulsations stop. All evolutionary models predict a much cooler red edge than the coolest ZZ Ceti observed, even though Wu \& Goldreich (1999) propose that pulsation should stop for periods around $1400 \mathrm{~s}$ (as observed) in their models of convection-dominated DA pulsations. Kanaan et al. (2002) looked for low-amplitude pulsators at the red edge, which would represent when the $\mathrm{ZZ}$ Cetis stop to pulsate, but they found none. Mukadam et al. (2006) found seven low amplitude pulsators with long periods, which is too large a number to be explained by inclination effects, especially when we consider that different $m$ 's cancel at opposite inclination angles. In our sample, WDJ 0303-0808, shows long periods and low amplitude.

Another reason for low amplitudes occurs for high mass stars, when their interiors are partially crystallized, like for BPM 37093 (Kanaan et al. 2005), as the pulsations are restricted to the upper layers above the crystallized core. However, to test this hypothesis for the low amplitude long period star observed, it is necessary to obtain spectra with higher $S N R$ than the SDSS spectra, specially in the region of the lines H8 and H9, because they are more sensitive to gravity, so measuring $T_{\text {eff }}$ and $\log g$ accurately. 
Mukadam et al. (2006), following Clemens (1993), discuss the relationship between weighted mean period and spectroscopic temperature, after studying the changes in pulsation properties across the ZZ Ceti instability strip. They found a linear relation between these two physical quantities for all ZZ Ceti stars with available temperatures.

Among the new pulsators, the two stars classified as NOVs are low amplitude pulsators, but they have completely different main periodicities. WDJ 0303-0808's detected periodicities are 707 and $1128 \mathrm{~s}$, typical of the red edge cool pulsator, while WDJ 0853+0005's main mode is around $264 \mathrm{~s}$, typical of a blue edge pulsator. Either WDJ 0303-0808 is a high mass star, where crystallization already occurs in its interior or it is an example of a pulsator stopping to pulsate. In Table 2, we listed $T_{\text {eff }}$ and $\log g$ derived from SDSS spectrum. Using these values and the radius calculated by Althaus et al. (2005), we derived a stellar mass of $0.93 M_{\odot}$. Kepler et al. (2006) derived a lower stellar mass of $0.83 M_{\odot}$ and $0.52 M_{\odot}$ from Gemini spectra fitting with LPT and whole spectra, respectively. As crystallization is important within the ZZ Ceti instability strip only for stars with masses higher than $\sim 1 M_{\odot}$, when a significant portion of the star will be crystallized, this star is most likely an example of a star stopping to pulsate. On the other hand, the high $\log g$ determined from SDSS spectra would indicate that the star WDJ 1650+3010 could be crystallized; however, its observed amplitude does not indicate that. The known correlation between $T_{\text {eff }}$ and $\log g$ determination could again explain this issue, as the determinations were based on low $S N R$ spectra.

Using the Mukadam et al. (2006) relation, the $T_{\text {eff }}$ for the star WDJ $0853+0005$ should be $11700 \mathrm{~K}$ in agreement with the one derived from the low $S N R$ spectra $11700 \mathrm{~K}$. For WDJ 0303-0808, the pulsation periods indicate $11000 \mathrm{~K}$, compared with $11400 \mathrm{~K}$ from the spectra. More observations of the star WDJ 0303-0808 are required, both with high SNR spectroscopy and time-resolved photometry, for a better understanding of its structure.

\section{Concluding remarks}

We report the discovery of seven new pulsating DA stars, bringing the total to 126 known $\mathrm{ZZ}$ Cetis in the narrow temperature range $12270 \mathrm{~K} \geq T_{\text {eff }} \geq 10850 \mathrm{~K}$, corresponding to the existence of a partial ionization of hydrogen and development of a subsurface convection zone. As two of these stars were previously reported as NOV, we need to lower the detection limits for all other NOVs observed by Mukadam et al. (2004) and Mullally et al. (2005), map the instability strip in both $T_{\text {eff }}$ and $\log g$ using accurate determinations of these parameters for the new variables and NOVs, for which we need higher SNR than those achieved in the SDSS spectra.

Acknowledgements. The authors acknowledge the support of CNPq fellowships and a NASA origins grant.

\section{References}

Althaus, L. G., García-Berro, E., Isern, J., \& Córsico, A. H. 2005, A\&A, 441, 689

Arras, P., Townsley, D. M., \& Bildsten, L. 2006, ApJ, 643, L119

Bergeron, P., Fontaine, G., Billères, M., Boudreault, S., \& Green, E. M. 2004, ApJ, 600, 404

Bradley, P. A. 1996, ApJ, 468, 350

Clemens, J. C. 1993, Baltic Astron., 2, 407

Eisenstein, D. J., Liebert, J., Harris, H. C., et al. 2006, ApJS, 167, 40

Finley, D. S., Koester, D., \& Basri, G. 1997, ApJ, 488, 375

Fontaine, G., Bergeron, P., Brassard, P., Billères, M., \& Charpinet, S. 2001, ApJ, 557,792

Gianninas, A., Bergeron, P., \& Fontaine, G. 2005, ApJ, 631, 1100

Kanaan, A., Kepler, S. O., Giovannini, O., \& Diaz, M. 1992, ApJ, 390, L89

Kanaan, A., Kepler, S. O., \& Winget, D. E. 2002, A\&A, 389, 896

Kanaan, A., Nitta, A., Winget, D. E., et al. 2005, A\&A, 432, 219

Kepler, S. O., Giovannini, O., Wood, M. A., et al. 1995, ApJ, 447, 874

Kepler, S. O., Castanheira, B. G., Saraiva, M. F. O., et al. 2005, A\&A, 442, 629

Kepler, S. O., Castanheira, B. G., Costa, A. F. M., \& Koester, D. 2006, MNRAS, 372,1799

Kleinman, S. J., Nather, R. E., Winget, D. E., et al. 1998, ApJ, 495, 424

Kleinman, S. J., Harris, H. C., Eisenstein, D. J., et al. 2004, ApJ, 607, 426

Mukadam, A. S., Mullally, F., Nather, R. E., et al. 2004, ApJ, 607, 982

Mukadam, A. S., Montgomery, M. H., Winget, D. E., Kepler, S. O., \& Clemens, J. C. 2006, ApJ, 640, 956

Mullally, F., Thompson, S. E., Castanheira, B. G., et al. 2005, ApJ, 625, 966

Nather, R. E., \& Mukadam, A. S. 2004, ApJ, 605, 846

Robinson, E. L., Kepler, S. O., \& Nather, R. E. 1982, ApJ, 259, 219

Wood, M. A. 1995, LNP White Dwarfs, 443, 41

Wu, Y., \& Goldreich, P. 1999, ApJ, 519, 783 